\title{
BMJ Open Does non-invasive brain stimulation modify hand dexterity? Protocol for a systematic review and meta-analysis
}

\author{
Anthony Terrence O'Brien, ${ }^{1}$ Gabriel Torrealba Acosta, ${ }^{1}$ Rodrigo Huerta, ${ }^{2}$ \\ Aurore Thibaut ${ }^{1,3}$
}

To cite: O'Brien AT, Torrealba Acosta G, Huerta R, et al. Does non-invasive brain stimulation modify hand dexterity? Protocol for a systematic review and meta-analysis. BMJ Open 2017;7:e015669. doi:10.1136/ bmjopen-2016-015669

- Prepublication history for this paper is available online. To view these files please visit the journal online (http://dx.doi org/10.1136/bmjopen-2016015669).

Received 21 December 2016 Revised 10 April 2017 Accepted 25 April 2017

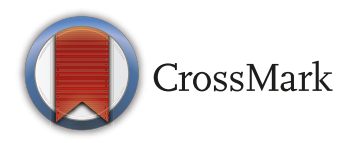

${ }^{1}$ Department of Physical Medicine and Rehabilitation, Spaulding Rehabilitation Hospital, Harvard Medical School, Charlestown, Massachusetts, USA ${ }^{2}$ Department of Medicine, Universidad Nacional Autonoma de Mexico, Coyoacan, Mexico ${ }^{3}$ Coma Science Group, GIGAConsciousness, University and University Hospital of Liège, Liège, Belgium

Correspondence to Dr Anthony Terrence 0'Brien; at.obrien39@gmail.com, aobrien@partners.org

\section{ABSTRACT}

Introduction Dexterity is described as coordinated hand and finger movement for precision tasks. It is essential for day-to-day activities like computer use, writing or buttoning a shirt. Integrity of brain motor networks is crucial to properly execute these fine hand tasks. When these networks are damaged, interventions to enhance recovery are frequently accompanied by unwanted side effects or limited in their effect. Non-invasive brain stimulation (NIBS) are postulated to target affected motor areas and improve hand motor function with few side effects. However, the results across studies vary, and the current literature does not allow us to draw clear conclusions on the use of NIBS to promote hand function recovery. Therefore, we developed a protocol for a systematic review and meta-analysis on the effects of different NIBS technologies on dexterity in diverse populations. This study will potentially help future evidence-based research and guidelines that use these NIBS technologies for recovering hand dexterity. Methods and analysis This protocol will compare the effects of active versus sham NIBS on precise hand activity. Records will be obtained by searching relevant databases. Included articles will be randomised clinical trials in adults, testing the therapeutic effects of NIBS on continuous dexterity data. Records will be studied for risk of bias. Narrative and quantitative synthesis will be done. Ethics and dissemination No private health information is included; the study is not interventional. Ethical approval is not required. The results will be reported in a peerreview journal.

Registration details PROSPERO International prospective register of systematic reviews registration number: CRD42016043809.

\section{INTRODUCTION}

The hand's somatotopy is extensively represented in the human motor cortex. ${ }^{12}$ Phylogenetically, this relates to the development of corticomotoneuronal cells that specialise in creating patterns of muscle activity that synergises into highly skilled movements. ${ }^{3}$ This organised hand-and-finger movement to use objects during a specific task is known as dexterity. ${ }^{4}$ Evolutionary, dexterity played a pivotal role in human survival and

\section{Strengths and limitations of this study}

This is a novel systematic review and meta-analysis focusing specifically on dexterity.

- We use continuous data not dependent on the evaluator or participant.

- This work will potentially help future evidence-based research and guidelines to refine non-invasive brain stimulation.

is fundamental to actives of daily living, and hence quality of life. ${ }^{56}$

This precision motor movement relies on integration of information from the cerebral cortex, the spinal cord, several neuromusculoskeletal systems and the external world to coordinate finger force control, finger independence, timing and sequence performance. ${ }^{7}$ During these tasks, multivoxel pattern decoding shows bilateral primary motor cortex activation (M1), which was responsible for muscle recruitment timing and hand movement coordination. ${ }^{89}$ This is related to motor cortex connectivity through the corpus callosum, to motor regions of the cerebellum and white matter integrity. ${ }^{10-15}$ Adequate motor output translates into successfully executed tasks, like picking up objects, turning over cards, manipulating cutlery, writing, using computer-hand interfaces like smartphones, playing an instrument and performing many other similarly precise skills. ${ }^{16}$

These motor tasks are negatively impacted when motor output networks are affected, as seen in stroke or Parkinson's disease. ${ }^{17} 18$ Therapeutic interventions that restore these damaged motor networks can be vital to restore fine motor movement after injury occurs. Pharmaceutical approaches often lead to adverse effects such as dyskinesias in Parkinson's disease. Moreover, even after intensive rehabilitation programmes, only about $5 \%-20 \%$ of patients with stroke fully 
recover their motor function. ${ }^{19-21}$ Non-invasive brain stimulation (NIBS) techniques, like transcranial direct current stimulation (tDCS) and repetitive transcranial magnetic stimulation (rTMS), are proposed adjuvant or stand-alone interventions to target these affected areas and improve fine motor function. ${ }^{22}{ }^{23}$ Briefly, these NIBS interventions are shown to influence the nervous system's excitability and modulate long-term plasticity, which may be beneficial to the brain's recovery of functions after injury. ${ }^{24-27}$

Fine hand motor ability is not studied as much in previous reviews of NIBS. Specifically, one narrative review focuses on rTMS in affected hand recovery poststroke; however, it does not consider the implications of varying International Classification of Functioning, Disability and Health (ICF) domains, data types and rater dependent outcomes, and its interpretability is limited without quantitative synthesis. ${ }^{28-31}$ The overarching conclusion was supportive of rTMS for paretic hand recovery, though with limited data to support its regular use, and a pressing need to study individualised patient parameters. ${ }^{28}$ One meta-analysis had positive and significant results when specifically studying the effects of rTMS on finger coordination and hand function after stroke. ${ }^{32}$ However, while various meta-analysis, and another systematic review, studied upper-limb movement after NIBS in distinct populations, they did not focus on precise hand function, pooled upper-limb outcomes with hand outcomes and presented mixed results. ${ }^{33-38}$

Motivated by this gap in the evidence for NIBS in dexterity, we will do a systematic review and meta-analysis of the literature on these brain stimulation technologies using outcomes that focus exactly on manual dexterity. These outcomes will be continuous and not dependent on the participant's or rater's observation (ie, they will be measured in seconds, or number of blocks/pegs placed, and not by an individual's interpretation). They will be comprised of multiple domains as defined by the ICF, providing an appreciation of function rather than only condition or disease. ${ }^{29-31}$ By focusing on the ICF model, we will be able to study dexterity across a larger sample of studies, NIBS techniques and conditions in order to provide a better understanding of brain stimulation efficacy on hand function in various populations.

\section{METHODS}

\section{General statement}

We followed the Preferred Reporting Items for Systematic Reviews and Meta-Analyses Protocols (PRISMA-P). ${ }^{39} 40$

\section{Search strategy and eligibility criteria}

Using the strategy in table 1 we will obtain records from PubMed, EMBASE, Web of Science, SciELO and OpenGrey. Eligible records will be focused on adult humans, enrolled in randomised clinical trials with primary or secondary outcomes for continuous manual dexterity data (box and block test, nine-hole peg test, Purdue pegboard test, Jebsen Taylor and functional dexterity test) in which sole or combined NIBS intervention (tDCS/TMS, transcranial alternative current stimulation, transcranial pulsed stimulation and transcranial random noise stimulation) trials are being studied therapeutically. Records will be excluded if they do not match the inclusion criteria and have at least one of the following characteristics: (1) non-randomised clinical trials; (2) observational studies; (3) case series/reports; (4) non-blinded studies (eg, open-label); (5) studies that do not have results for at least one of the continuous manual dexterity instruments being extracted (box and block test, nine-hole peg test, Purdue pegboard test, Jebsen Taylor and functional dexterity test); (6) studies that do not use at least one of the previously brain stimulation interventions therapeutically; and (7) studies not published in English/Spanish/ French.

\section{Screening and selection of records}

Two independent researchers will screen the records from the publication's title and abstract. A Cochrane recommended web and mobile application, Rayyan, will be used to ensure that the screening process is blinded between the two independent researchers. ${ }^{41} \mathrm{~A}$ third researcher will resolve disagreements among screened records. Three independent researchers will assess the records' eligibility, which will be cross-validated within the group. This will be documented on a modified spreadsheet by The University of Texas Health Center at Houston for studying eligibility. ${ }^{42}$ After which the references of included records will be reviewed, screened and undergo the same inclusion/exclusion process. The results of the search strategy, screening and eligibility will be summarised into a PRISMA Flow Diagram. ${ }^{40}$

\section{Assessment of risk of bias in included studies}

Risk of bias will be assessed using the Cochrane Collaboration tool for assessing the risk of bias (table 8.4a of the Cochrane Collaboration Handbook for Systematic Reviews of Interventions). ${ }^{43}$ The following elements will be reviewed and recorded: (1) random sequence generation; (2) allocation concealment; (3) blinding of participants and personnel; (4) blinding of outcome assessment; (5) incomplete outcome data; and (6) selective reporting. For each record, each item will be judged as low, unclear or high risk as set out in by Green and Higgins. ${ }^{43}$ Three independent researchers will perform the risk of bias review, with cross-validation within the group. We will use custom checklists generated in Microsoft Office Excel 2007 (V.12.0) to capture data. These data will be incorporated into tables, figures, graphs and descriptive narrative, which will guide evaluation of the records' internal validity.

\section{Data extraction}

Data will be extracted into a custom spreadsheet that will incorporate at the following: (1) bibliographic details of primary paper (author, title of study, year and journal); (2) demographics (number of participants, age and gender); (3) clinical information (eg, diagnosis and 


\begin{tabular}{|c|c|c|}
\hline $\begin{array}{l}\text { Search } \\
\text { engine }\end{array}$ & Search query & $\begin{array}{l}\text { Date of } \\
\text { search }\end{array}$ \\
\hline Embase & $\begin{array}{l}\text { (box AND block AND test) OR (nine AND hole AND peg AND test) OR (purdue AND pegboard AND } \\
\text { test) OR (functional AND dexterity AND test) OR (Jebsen) AND (test) AND (transcranial AND direct } \\
\text { AND current AND stimulation OR tdcs OR tms OR tpcs OR tacs OR (transcranial AND magnetic } \\
\text { AND stimulation) OR (transcranial AND pulsed AND stimulation) OR (transcranial AND alternating } \\
\text { AND current AND stimulation) OR (random AND noise) OR (transcranial AND magnetic AND } \\
\text { stimulation) OR nibs OR (non AND invasive AND brain AND stimulation) OR (brain AND stimulation)) } \\
\text { AND ('randomized controlled trial'/exp OR 'controlled clinical trial'/exp OR randomized:ab,ti OR } \\
\text { placebo:ab,ti OR randomly:ab,ti OR trial:ab,ti OR groups:ab,ti) }\end{array}$ & 1-Aug-16 \\
\hline $\begin{array}{l}\text { Web of } \\
\text { Science }\end{array}$ & $\begin{array}{l}\text { (manual dexterity or motor movement or motor skills) AND TOPIC: (box block test OR nine hole } \\
\text { peg test OR functional pegboard test OR purdue pegboard test OR functional dexterity test OR } \\
\text { Jebsen Test) AND TOPIC: (transcranial direct current stimulation OR tdcs OR transcranial magnetic } \\
\text { stimulation OR tms OR NIBS or non invasive brain stimulation OR brain stimulation OR tpcs OR } \\
\text { tacs OR transcranial pulsed current stimulation OR transcranial alternating current stimulation OR } \\
\text { random noise) }\end{array}$ & 1-Aug-16 \\
\hline
\end{tabular}

The PubMed and Embase search queries include the 'Highly Sensitive Search Strategy' designed by the Cochrane Collaboration, which yield a sensitivity of $98 \%$ and precision of $13 \%$ for randomised clinical trials. ${ }^{4353 *}$ The search strategy for ScieLo was also modified to Spanish accordingly.

diagnostic criteria, medications, therapy, adverse events, comorbidities, inclusion and exclusion criteria for individual studies, type of lesion and extension and time since onset in stroke); (4) trial characteristics (trial design, duration, number of follow-ups, groups and limitations reported by authors); (5) parameters of NIBS will be noted (eg, montage, site of stimulation, current intensity, electrode size, current density, duration of stimulation, polarity, number of pulses, trains, sessions, frequency of pulse, duration of pulse, number of pulses per train, number of trains, number of pulses per session, total number of pulses delivered and \% RMT); (6) the primary and secondary outcomes measures; (7) the results of the continuous manual dexterity activity outcomes (box and block test, nine-hole peg test, Purdue pegboard test, Jebsen Taylor hand function test and functional dexterity test); and (8) funding source and conflict of interest.
These data will be organised into tables, figures, graphs and descriptive narrative. All spreadsheets in this review will be piloted in a sample of two records prior to use.

\section{Data synthesis}

We anticipate the confines of this approach will parallel former systematic reviews and meta-analysis on upperlimb function; for example, there will be heterogeneous study design, pathophysiological variability like different brain architecture between chronic and acute conditions, parameters for stimulation will vary, alternate trial designs will be present and even outcomes will be recorded through different instruments. ${ }^{28}$ 32-38 44-48 For this reason, a random effects assumption will be assumed for measuring the pooled data. That is, we expect the true effect to differ from study to study. In regards to the outcome, even though the selected outcomes objectively 
measure improvement in manual dexterity on a continuous scale, we expect the scale will differ from study to study, and it will not be meaningful to combine raw means. ${ }^{49}$ Therefore, we will divide the mean difference of each study by the study's SD to create a comparable index. Prescores and post scores will be used and also compared against real versus sham conditions.

Using Stata V.13 ME $\left({ }^{50}\right.$ Stata Statistical Software: Release 13), we will combine the records' effect size and present this information graphically via a forest plot, with the estimated effect size, $95 \%$ CI an $015642 \mathrm{~d} p$ value.${ }^{50}$ Heterogeneity will be assessed by means of Q-statistics and $\mathrm{I}^{2}$. Metabias will be evaluated with Begg's funnel plots. Sensitivity analysis based on pathology, NIBS technique and risk of bias will be done.

\section{Dealing with missing data}

When data are unclear or missing, we will attempt to contact authors for data. However, when not successful, we will use available data from record where possible.

\section{Summary of findings table}

We will organise a summary of findings table based on chapter 11 of the Cochrane Handbook for Systematic Reviews of Interventions. ${ }^{43}$ The main dexterity outcomes studied will be pooled and also looked at individually. The body of evidence will be reviewed with the Grading of Recommendations Assesment, Development and Evaluation (GRADE) system. ${ }^{51}$

\section{ETHICS AND DISSEMINATION}

No private health information will be included, and the study is not interventional. Therefore, ethical approval is not required. The results will be reported in an international peer-reviewed journal. Furthermore, we will attempt to publish the findings in an open-label peer-reviewed journal.

\section{PROTOCOL AMENDMENTS}

Protocol deviations will be noted and explained in the final published product. Also, amendments will be noted in the corresponding PROSPERO registry. ${ }^{52}$

Correction notice This paper has been amended since it was published Online First. Owing to a scripting error, some of the publisher names in the references were replaced with 'BMJ Publishing Group'. This only affected the full text version, not the PDF. We have since corrected these errors and the correct publishers have been inserted into the references.

Acknowledgements The authors would like to thank Thea Villate Bocconello for editing and proof-reading this manuscript.

Contributors ATO conceptualised the protocol and made substantial contributions to the design, drafting and revision of the work. GTA, RH and AT significantly contributed to designing and critically reviewing the protocol. All authors approved the final version of the manuscript and assume accountability for all aspects of the work.

Funding This review was funded by the Harvard Open-Access Publishing Equity (HOPE) fund and the author's final version is deposited in the Digital Access to Scholarship at Harvard (DASH). Other than reimbursing the cost of reasonable article processing fees, the mentioned institution was not directly responsible for any development of the protocol.
Competing interests The authors state that they have no significant competing financial, professional or personal interests that might have influenced the performance or presentation of the work described in this manuscript.

Patient consent Not applicable.

Provenance and peer review Not commissioned; externally peer reviewed.

Data sharing statement We plan on making data used for analysis openly available after publication. We will attempt to publish data in open access journals and eventually data repositories.

Open Access This is an Open Access article distributed in accordance with the Creative Commons Attribution Non Commercial (CC BY-NC 4.0) license, which permits others to distribute, remix, adapt, build upon this work non-commercially, and license their derivative works on different terms, provided the original work is properly cited and the use is non-commercial. See: http://creativecommons.org/ licenses/by-nc/4.0/

(c) Article author(s) (or their employer(s) unless otherwise stated in the text of the article) 2017. All rights reserved. No commercial use is permitted unless otherwise expressly granted.

\section{REFERENCES}

1. Hlustík P, Solodkin A, Gullapalli RP, et al. Somatotopy in human primary motor and somatosensory hand representations revisited. Cereb Cortex 2001;11:312-21.

2. Metman LV, Bellevich JS, Jones SM, et al. Topographic mapping of human motor cortex with transcranial magnetic stimulation: homunculus revisited. Brain Topogr 1993;6:13-19 .

3. Rathelot JA, Strick PL. Subdivisions of primary motor cortex based on cortico-motoneuronal cells. Proc Natl Acad Sci U S A 2009;106:918-23.

4. Backman C, Gibson SCD, Parsons J. Assessment of hand function: the relationship between Pegboard Dexterity and Applied Dexterity. Can J Occup Ther 1992;59:208-13.

5. Dollar AM. Classifying human hand use and the activities of daily living. Springer Tracts Adv Robot 2014;95:201-16.

6. Young RW. Evolution of the human hand : the role of throwing and. $J$ Anat 2007;202:1-14.

7. Makofske B, Dexterity M, Kreutzer JS, et al. Encyclopedia of Clinical Neuropsychology. New York, NY: Springer New York, 2011:1522.

8. Di Bono MG, Begliomini C, Castiello U, et al. Probing the reachinggrasping network in humans through multivoxel pattern decoding. Brain Behav 2015;5.

9. Davare M, Duque J, Vandermeeren Y, et al. Role of the ipsilateral primary motor cortex in controlling the timing of hand muscle recruitment. Cereb Cortex 2007;17:353-62.

10. Steele CJ, Scholz J, Douaud G, et al. Structural correlates of skilled performance on a motor sequence task. Front Hum Neurosci 2012;6:289.

11. Tuch DS, Salat DH, Wisco JJ, et al. Choice reaction time performance correlates with diffusion anisotropy in white matter pathways supporting visuospatial attention. Proc Natl Acad Sci U S A 2005;102:12212-7.

12. Johansen-Berg $H$, Della-Maggiore $V$, Behrens TE, et al. Integrity of white matter in the corpus callosum correlates with bimanual coordination skills. Neuroimage 2007;36(Suppl 2):T16-T21.

13. Della-Maggiore $\mathrm{V}, \mathrm{Scholz} \mathrm{J}$, Johansen-Berg $\mathrm{H}$, et al. The rate of visuomotor adaptation correlates with cerebellar white-matter microstructure. Hum Brain Mapp 2009;30:4048-53.

14. Mercado R, Constantoyannis C, Mandat T, et al. Expectation and the placebo effect in Parkinson's disease patients with subthalamic nucleus deep brain stimulation. Mov Disord 2006;21:1457-61.

15. Tomassini V, Jbabdi S, Kincses ZT, et al. Structural and functional bases for individual differences in motor learning. Hum Brain Mapp 2011;32:494-508.

16. Dexterity M, Kreutzer JS, et al. Encyclopedia of Clinical Neuropsychology somatotopy is extensively rep. 3. eds. New York: NY Springer New York, 2011.

17. Massano J, Bhatia KP. Clinical approach to Parkinson's disease: features, diagnosis, and principles of management. Cold Spring Harb Perspect Med 2012;2:a008870-15.

18. Lang CE, Bland MD. Assessment of Upper Extremity impairment and Activity Following Stroke: Foundations for Clinical Decision Making. 2014;26:104-15.

19. Kwakkel G, Kollen BJ, van der Grond J, et al. Probability of regaining dexterity in the Flaccid Upper Limb in acute stroke. Stroke 2003;34:2181-6. 
20. Kwakkel G, Kollen BJ. Predicting activities after stroke: what is clinically relevant? Int J Stroke 2013;8:25-32.

21. Olanow CW, Koller WC. An algorithm (decision tree) for the management of Parkinson's disease: treatment guidelines. Neurology 1998;50:S1

22. Lefaucheur JP, André-Obadia N, Antal A, et al. Evidence-based guidelines on the therapeutic use of repetitive transcranial magnetic stimulation (rTMS). Clin Neurophysiol 2014;125:2150-206.

23. Lefaucheur JP, Antal A, Ayache SS, et al. Evidence-based guidelines on the therapeutic use of transcranial direct current stimulation (tDCS). Clin Neurophysiol 2017;128:56-92.

24. Monte-Silva K, Kuo MF, Hessenthaler S, et al. Induction of late LTPlike plasticity in the human motor cortex by repeated non-invasive brain stimulation. Brain Stimul 2013;6:424-32.

25. Rushmore RJ, DeSimone C, Valero-Cabré A. Multiple sessions of transcranial direct current stimulation to the intact hemisphere improves visual function after unilateral ablation of visual cortex. Eur J Neurosci 2013;38:3799-807.

26. Nitsche MA, Cohen LG, Wassermann EM, et al. Transcranial direct current stimulation: state of the art 2008. Brain Stimul 2008;1:206-23.

27. Monte-Silva K, Kuo MF, Liebetanz D, et al. Shaping the optimal repetition interval for cathodal transcranial direct current stimulation (tDCS). J Neurophysiol 2010;103:1735-40.

28. Lüdemann-Podubecká J, Bösl K, Nowak DA. Repetitive transcranial magnetic stimulation for motor recovery of the upper limb after stroke. Prog Brain Res 2015;218:281-311.

29. WHO. Icf checklist. Organization, 2003:1-15.

30. Kostanjsek N. Use of the International classification of functioning, disability and Health (ICF) as a conceptual framework and common language for disability statistics and health information systems. BMC Public Health 2011;11(Suppl 4):S3.

31. Stucki G, Kostanjsek N, Ustün B, et al. ICF-based classification and measurement of functioning. Eur $J$ Phys Rehabil Med 2008;44:315-28.

32. Le Q, Qu Y, Tao Y, et al. Effects of repetitive transcranial magnetic stimulation on hand function recovery and excitability of the motor cortex after stroke: a meta-analysis. Am J Phys Med Rehabil 2014;93:422-30.

33. Hao Z, Wang D, Zeng Y, et al. Repetitive transcranial magnetic stimulation for improving function after stroke. Cochrane Database Syst Rev 2013:CD008862.

34. Tedesco Triccas L, Burridge JH, Hughes AM, et al. Multiple sessions of transcranial direct current stimulation and upper extremity rehabilitation in stroke: a review and meta-analysis. Clin Neurophysiol 2016;127:946-55.

35. Kang N, Summers JJ, Cauraugh JH. Transcranial direct current stimulation facilitates motor learning post-stroke: a systematic review and meta-analysis. J Neurol Neurosurg Psychiatry 2016;87:345-55.

36. Fregni F, Simon DK, Wu A, et al. Non-invasive brain stimulation for Parkinson's disease: a systematic review and meta-analysis of the literature. J Neurol Neurosurg Psychiatry 2005;76:1614-23.

37. Elsner B, Kugler J, Pohl M, et al. Transcranial direct current stimulation (tDCS) for improving function and activities of daily living in patients after stroke. Cochrane Database Syst Rev 2013:CD009645.

38. Pollock A, Farmer SE, Brady MC, et al. Interventions for improving upper limb function after stroke. Cochrane Database Syst Rev 2014:CD010820.

39. Shamseer L, Moher D, Clarke M, et al. Preferred reporting items for systematic review and meta-analysis protocols (PRISMA-P) 2015: elaboration and explanation. BMJ 2015;349:g7647.

40. Moher D, Shamseer L, Clarke M, et al. Preferred reporting items for systematic review and meta-analysis protocols (PRISMA-P) 2015 statement. Syst Rev 2015;4:1.

41. Ouzzani M, Hammady H, Fedorowicz Z, et al. Rayyan-a web and mobile app for systematic reviews. Syst Rev 2016;5:210.

42. VonVille H. Excel Workbooks for Systematic Reviews \& corresponding handouts: excel Workbooks for SRs. Univ. Texas. Heal. Sci. Cent. Houst. http://libguides.sph.uth.tmc.edu/excel_ workbook home/excel SR workbook.

43. Higgins JPT, Green S. The Cochrane Collaboration. Table 7.7.a: Formulae for combining groups. Cochrane Handbook for Systematic Reviews of Interventions Version 5.1.0 (updated March 2011), 2011.

44. O'Brien AT, Amorim R, Rushmore RJ, et al. Motor Cortex Neurostimulation Technologies for chronic Post-stroke pain: implications of tissue damage on stimulation currents. Front Hum Neurosci 2016;10.

45. Bastani $A$, Jaberzadeh $S$. Does anodal transcranial direct current stimulation enhance excitability of the motor cortex and motor function in healthy individuals and subjects with stroke: a systematic review and meta-analysis. Clin Neurophysiol 2012;123:644-57.

46. Butler AJ, Shuster M, O'Hara E, et al. A meta-analysis of the efficacy of anodal transcranial direct current stimulation for upper limb motor recovery in stroke survivors. $J$ Hand Ther 2013;26:162-71.

47. Kang N, Summers JJ, Cauraugh JH. Transcranial direct current stimulation facilitates motor learning post-stroke: a systematic review and meta-analysis. J Neurol Neurosurg Psychiatry 2016;87:345-55.

48. Zhu H, Lu Z, Jin Y, et al. Low-frequency repetitive transcranial magnetic stimulation on Parkinson motor function: a meta-analysis of randomised controlled trials. Acta Neuropsychiatr 2015;27:82-9.

49. Borenstein M, Hedges L V, Higgins JPT, et al. Introduction to MetaAnalysis. Psychother Res J Soc Psychother Res 2009;19:421.

50. StataCorp. Stata Statistical Software: release 13. 2013. 2013.

51. Schünemann H, Brożek J, Guyatt G, et al. GRADE handbook for grading quality of evidence and strength of recommendations. GRADE Work. Gr, 2013. http://tech.cochrane.org/gradepro

52. O'Brien AT, Huerta R, Torrealba G, et al. Does non-invasive brain stimulation improve manual dexterity: a systematic review and meta-analysis. PROSPERO 2016 CRD42016043809. 2016. http://www.crd.york.ac.ukPROSPERO/display_record.asp?ID= CRD42016043809

53. McKibbon KA, Wilczynski NL, Haynes RB. Retrieving randomized controlled trials from medline: a comparison of 38 published search filters. Health Info Libr J 2009;26:187-202. 